\title{
Species Composition and Diversity of Insects of the Kogyae Strict Nature Reserve in Ghana
}

\author{
Rosina Kyerematen ${ }^{1,2 *}$, Erasmus Henaku Owusu1, Daniel Acquah-Lamptey ${ }^{1}$, \\ Roger Sigismund Anderson ${ }^{2}$, Yaa Ntiamoa-Baidu 1,3 \\ ${ }^{1}$ Department of Animal Biology and Conservation Science, University of Ghana, Legon, Ghana \\ ${ }^{2}$ African Regional Postgraduate Programme in Insect Science, University of Ghana, Legon, Ghana \\ ${ }^{3}$ Centre for African Wetlands, University of Ghana, Legon, Ghana \\ Email: rkyerematen@ug.edu.gh, dacquahlamptey@gmail.com, erasmus67@yahoo.com, \\ sigiandy@hotmail.com, ynbaidu@ug.edu.gh
}

Received 6 September 2014; revised 9 November 2014; accepted 21 November 2014

Copyright (C) 2014 by authors and Scientific Research Publishing Inc.

This work is licensed under the Creative Commons Attribution International License (CC BY). http://creativecommons.org/licenses/by/4.0/

(c) (i) Open Access

\begin{abstract}
Kogyae Strict Nature Reserve, the only one in Ghana, was established to promote scientific research, particularly on how nature revitalizes itself after major disasters, and also to check the southward drift of the savannah grassland. This study presents the first comprehensive inventory of species composition and diversity of insects of the Reserve. Insects were surveyed between September 2011 and June 2012 to capture the end of the rainy season, the dry season and the peak of the wet season. Samples were taken from two sites within the Reserve, Dagomba and Oku using various sampling techniques including pitfall traps, malaise traps and sweep nets. Insect communities were characterized in terms of, 1) species richness estimators, 2) species richness, 3) Shannon-Weiner Index of Diversity, 4) Pielou's evenness and 5) Bray-Curtis similarity. A total of 8147 individuals representing 135 families from 21 orders were recorded. This included 107 species of butterflies from 9 families and 20 species of dragonflies from 3 families. Oku recorded the highest species numbers $(S=63)$ and richness $(d=12.16)$ with a high evenness of species $U=$ $0.9377)$ during the peak of the wet season; and the lowest species numbers $(S=58)$ and Margalef's index of $(d=10.14)$ in January. The highest Shannon diversity index of $(H=3.927)$ was recorded at Dagomba in January.
\end{abstract}

\section{Keywords}

Insects, Diversity, Kogyae Strict Nature Reserve, Species Richness

\footnotetext{
${ }^{*}$ Corresponding author.
}

How to cite this paper: Kyerematen, R., Owusu, E.H., Acquah-Lamptey, D., Anderson, R.S. and Ntiamoa-Baidu, Y. (2014) Species Composition and Diversity of Insects of the Kogyae Strict Nature Reserve in Ghana. Open Journal of Ecology, 4, 1061-1079. http://dx.doi.org/10.4236/oje.2014.417087 


\section{Introduction}

The Kogyae Strict Nature Reserve (originally the Kujani Bush Forest Reserve) was designated by the Government of the colonial Gold Coast in 1952 and became a fully fledged Forest Reserve, in 1962 under the administration of the Forestry Department [1]. The main objective for designation of the reserve was to preserve the natural vegetation and animal life in the area, protect the head waters of the tributaries of the Sene and Afram rivers and check the downward expansion of the savanna zone. In 1971, the Wildlife Department (now Wildlife Division of the Ghana Forestry Commission) took over the Forest Reserve from the Forestry Department and designated it as a Strict Nature Reserve, devoted solely to scientific research, but still guided by the initial objectives.

The principal role of reserves is to separate elements of biodiversity from processes that threaten their existence in the wild and this is done within the constraints imposed by large and rapidly increasing numbers of human population in many parts of the world and their attendant requirement of space, materials and waste disposal [2].

Insects are used as indicators of environmental degradation as well as indicators of the ecological well-being of an ecosystem, hence the need for their conservation. Insects are critical natural resources in ecosystems, particularly those of forests [3]. In addition to their role as efficient pollinators and natural/biological pest control agents, some insect species are important indicators in ecosystems management [4]. Africa has a rich insect biodiversity; however, only few taxa have been collected extensively and well studied [5].

A European Union report [6] gives a detailed description of the mammals, amphibians and birds of Kogyae, but there has been no published study of the insects of the reserve, with the exception of butterflies [7].

This study therefore gives the first comprehensive inventory, showing species composition and diversity of the insects of the Kogyae Strict Nature Reserve. The inventory of the insect diversity and abundance was undertaken as a series of studies seeking to investigate the potential impacts of climate change on biodiversity in Ghana with special reference to species of conservation concern.

\section{Methods}

\subsection{Sampling Sites}

The Kogyae Strict Nature Reserve (KSNR) lies within the Guinean Forests of West Africa Biodiversity hotspot as defined by [8]. It is an IUCN category Ia reserve i.e. a protected area managed mainly for science. It covers an area of 33,618 hectares $\left(386 \mathrm{~km}^{2}\right)$ with an annual average rainfall of $1254 \mathrm{~mm}$. It lies at an altitude range of $80-210 \mathrm{~m}$ and located on latitude, longitude $7.12 \mathrm{~N}-1.11 \mathrm{~W}$ [9]. The Kogyae Strict Nature Reserve located in the Transitional Semi-Deciduous Forest Zone of Ghana with tracts of open woodland and grassland, lies $25 \mathrm{~km}$ south-east of Ejura and 50 km north-east of Mampong and boarders Kumawu and Kwamang traditional areas of the Ashanti Region. This reserve stretches along the Afram Plains of Ghana, which is a wide expanse of flat arable land. Geographically, this area separates the southern forest from the northern savanna regions [1]. Currently, four habitat types can be recognised within in the reserve, namely: 1) farmlands, including land that is presently under cultivation and those that have been recently abandoned; 2) grassland and woodland savannah, with scattered trees and varying canopies between $20 \%-70 \%$; 3 ) forest found along rivers and streams and the natural forest of the original Kujani Forest Reserve with canopy cover ranging from $50 \%$ to over $80 \%$; and 4 ) bare rock areas restricted to the southern and south-western end of the reserve where rock outcrops form a ridge.

Sampling was done in two distinct habitats types: 1) riparian forest mosaic at Oku Nkwanta; and 2) woodland savannah with some rocky outcrops at Dagomba Village towards Asasebonso.

\subsection{Sampling Methods}

Sampling was carried out using the following trapping techniques: malaise traps, flight interception traps, pitfall traps, Charaxes traps, yellow pan traps, butterfly/aerial nets, sweeping, visual observations and handpicking. One each of malaise and flight interception traps (which are designed to intercept insects in flight) were set up, at each sampling site to trap small flying, insects. Alcohol-loving butterflies and insects attracted to fermenting foods were sampled using the Charaxes traps, five of which were set up at each site. Rotten banana, mashed and mixed with alcohol (palm wine and or beer) was used as bait for the Charaxes traps; five pitfall traps were randomly set up at each site for crawling and ground dwelling insects; sweeping was done for vegetative-dwelling insects and five yellow pans were placed randomly at each site to collect mostly flying insects attracted to the 
yellow colour. The butterfly nets were used for butterflies and other high flying insects; visual observations and counts were done for dragonflies since they are very powerful and fast fliers and could not be captured using the Aerial nets and also for those insects that were missed by the nets and those insects that had already been captured since it was not necessary to capture more insects than necessary. Handpicking with forceps was done for insects in easy reach. A minimum of three hours was used for each sampling period twice each day for seven days using random walk sampling by three persons for each site. Insects collected were killed using a killing bottle containing ethyl acetate and kept either in alcohol or glassine envelops for later examination and identification.

Identification of insects was done with reference to the collection in the Museum of the Department of Animal Biology and Conservation Science, University of Ghana, [10]-[23].

Field collections were carried out in September 2011, January and June 2012, the end of the rainy season, the dry and peak of the rainy seasons respectively. Each season was sampled over a period of twelve days.

\subsection{Data Analyses}

In order to show how species are related in reference to their distribution, cluster analyses was performed on the data using Bray-Curtis similarity using PRIMER software 6.1.5 [24] [25]. Species contributing at least one percent of the total abundance at each sampling site were included in the analysis. The original data was fourth-root transformed and standardized in order to minimize the weighting of numerically dominant species. Species accumulation curves, species richness estimators [26]-[29] using Estimate S [27] as well as actual species diversity indices were computed and treated as surrogates for biodiversity [30].

The indices computed [31] were:

1) Species richness (Margalef): $d=(S-1) / \log (N)$-this is a measure of the number of species present, making some allowance for the number of individuals.

2) Pielou's evenness $J=H^{\prime} / \ln (S)$-this is a measure of equitability, a measure of how evenly the individuals are distributed among the different species.

3) Shannon-Wiener index $H^{\prime}=-\sum p_{i} \ln \left(p_{i}\right)$-incorporates both species richness and equitability components.

$n_{i}=$ the number of individuals of species $i$ in the sample, $\sum n_{i}=N . p_{i}=$ the proportion of individuals of species $i$ in the sample, $p_{i}=n_{i} / N . S=$ the number of species in the sample, $N=$ the total number of individuals in the sample.

\section{Results}

A total of 8147 individual insects belonging to 135 families and 21 orders were recorded in the survey. In this paper the butterflies and dragonflies are presented separately from all the other insects due to their conservation interest and the very important role they play as indicators of ecosystem health.

\subsection{Butterflies}

107 species of butterflies (Table 1) belonging to nine families were recorded during the survey: Nymphalidae, Acraedae, Satyridae, Papilionidae, Danaidae, Pieridae, Charaxidae, Lycaenidae, and Hesperidae, with the Nymphalidae dominating with 35 species followed by Pieridae with 27 species. Even though Lycaenidae had only 13 species, it was the most abundant with 871 individuals recorded. The Charaxidae recorded the lowest abundance of only 43 individuals. Euchrysops albistriata, and Hamanumida daedalus were the most abundant species of butterflies with 387 and 193 individuals respectively.

Table 2 shows a calculation of a suite of diversity indices and richness estimators for butterfly species collected from the two sampling sites, Dagomba (ED) and Oku (EO), at the Kogyae Strict Nature Reserve in September, 2011, January and June, 2012 (ED1, ED2, ED3 and EO1, EO2, EO3). Dagomba, (ED) generally recorded the highest species abundance and richness for all seasons. ED recorded the highest abundance $(N=807)$ and highest dominance (lowest evenness, $\left.J^{\prime}=0.7524\right)$ in June with the highest species numbers $(S=106)$ recorded in January which was corroborated by the highest Margalef index $(d)$ of 15.90 . The lowest abundance $(N=160)$ as well as species numbers $(S=58)$ was recorded at Oku $(E O)$ in June and January respectively although it had its highest Shannon Weiner diversity, $H$, of 3.885 in September. The relatively low evenness rec- 
Table 1. Diversity of butterfly species recorded within the Kogyae Strict Nature Reserve.

\begin{tabular}{|c|c|c|c|c|c|c|}
\hline \multirow{2}{*}{ SPECIES } & \multicolumn{3}{|c|}{ DAGOMBA } & \multicolumn{3}{|c|}{ OKU VILLAGE } \\
\hline & July & January & June & July & January & June \\
\hline Euriphene barombina & 1 & 11 & 2 & 0 & 4 & 2 \\
\hline Euryphene sp. & 0 & 3 & 0 & 0 & 1 & 0 \\
\hline Euphaedra sarcoptera & 0 & 1 & 1 & 0 & 0 & 1 \\
\hline Euphaedra edwardsi & 0 & 2 & 0 & 0 & 0 & 0 \\
\hline Euphaedra hapalyce & 0 & 3 & 0 & 0 & 0 & 0 \\
\hline Euphaedra eleus & 0 & 1 & 0 & 0 & 0 & 0 \\
\hline Euphaedra medon & 0 & 2 & 1 & 0 & 0 & 0 \\
\hline Euphaedra crockeri & 0 & 1 & 0 & 0 & 0 & 0 \\
\hline Euphaedra xypete & 0 & 3 & 0 & 0 & 0 & 0 \\
\hline Eurytela dryope & 0 & 2 & 1 & 0 & 0 & 0 \\
\hline Pseudoacrea lucretia & 0 & 2 & 1 & 0 & 0 & 0 \\
\hline Pseudoacrea sp. & 3 & 1 & 0 & 0 & 2 & 1 \\
\hline Euriphene feronia & 0 & 4 & 0 & 0 & 0 & 0 \\
\hline Neptis melicerta & 6 & 22 & 6 & 0 & 5 & 2 \\
\hline Neptis agatha & 4 & 9 & 3 & 0 & 1 & 2 \\
\hline Neptis metella & 3 & 18 & 6 & 0 & 2 & 4 \\
\hline Neptis morosa & 2 & 4 & 3 & 1 & 0 & 1 \\
\hline Salamis anacardii & 2 & 14 & 1 & 1 & 0 & 0 \\
\hline Salamis sp. & 0 & 8 & 2 & 0 & 0 & 0 \\
\hline Junonia terea & 0 & 1 & 9 & 2 & 1 & 1 \\
\hline Junonia oenone & 0 & 2 & 1 & 1 & 1 & 2 \\
\hline Precis octavia & 0 & 2 & 1 & 1 & 3 & 1 \\
\hline Gnophodes chelys & 0 & 1 & 2 & 1 & 0 & 0 \\
\hline Gnophodes betsimena & 0 & 2 & 1 & 0 & 0 & 0 \\
\hline Kallima rumia & 2 & 1 & 1 & 0 & 0 & 0 \\
\hline Phalanta phalantha & 3 & 15 & 17 & 2 & 1 & 3 \\
\hline Ariadne enotera & 0 & 1 & 2 & 0 & 0 & 0 \\
\hline Aterica galene & 0 & 3 & 0 & 0 & 0 & 0 \\
\hline Byblia achelola & 0 & 6 & 2 & 0 & 0 & 0 \\
\hline Hammanumida daedalus & 8 & 39 & 79 & 3 & 50 & 14 \\
\hline Melanitis leda & 0 & 0 & 1 & 0 & 0 & 0 \\
\hline Byblia sp. & 0 & 2 & 0 & 0 & 0 & 0 \\
\hline Hypolimnas salmacis & 5 & 1 & 3 & 3 & 1 & 2 \\
\hline Hypolimnas missipus & 3 & 2 & 2 & 2 & 0 & 1 \\
\hline
\end{tabular}




\section{Continued}

\begin{tabular}{|c|c|c|c|c|c|c|}
\hline Hypolimnas dinarcha & 2 & 1 & 2 & 1 & 0 & 0 \\
\hline Acraea ераеа & 0 & 4 & 3 & 1 & 1 & 1 \\
\hline Acraea zetes & 2 & 1 & 12 & 0 & 0 & 1 \\
\hline Acraea eponina & 0 & 3 & 1 & 0 & 11 & 3 \\
\hline Acraea sp. & 0 & 8 & 3 & 2 & 2 & 1 \\
\hline Hallelesis halyma & 3 & 9 & 0 & 1 & 0 & 0 \\
\hline Ypthimomorpha itonia & 1 & 2 & 0 & 1 & 0 & 0 \\
\hline Bicyclus zinebi & 2 & 12 & 7 & 2 & 3 & 1 \\
\hline Bicyclus safitza & 10 & 11 & 11 & 3 & 5 & 11 \\
\hline Bicyclus auricruda & 1 & 2 & 0 & 0 & 0 & 0 \\
\hline Bicyclus funebris & 3 & 2 & 0 & 1 & 1 & 2 \\
\hline Bicyclus sandace & 1 & 1 & 0 & 2 & 0 & 1 \\
\hline Bicyclus vulgaris & 2 & 1 & 1 & 1 & 0 & 1 \\
\hline Papilio demodocus & 3 & 32 & 9 & 2 & 2 & 5 \\
\hline Papilio dardanus & 0 & 1 & 2 & 1 & 0 & 3 \\
\hline Papilio nireus & 0 & 2 & 11 & 0 & 1 & 2 \\
\hline Papilio bromius & 0 & 1 & 2 & 0 & 0 & 0 \\
\hline Graphium policenes & 0 & 1 & 2 & 0 & 0 & 0 \\
\hline Amauris niavus & 0 & 3 & 1 & 4 & 31 & 3 \\
\hline Amauris egialea & 0 & 4 & 7 & 2 & 10 & 1 \\
\hline Amauris sp. & 0 & 2 & 2 & 1 & 6 & 0 \\
\hline Danaus chrysippus & 4 & 16 & 3 & 1 & 3 & 5 \\
\hline Tirumala petiverana & 2 & 9 & 13 & 3 & 1 & 1 \\
\hline Eurema brigitta & 6 & 12 & 21 & 4 & 9 & 6 \\
\hline Eurema senegalensis & 8 & 2 & 3 & 2 & 0 & 1 \\
\hline Eurema hecabe & 5 & 8 & 5 & 3 & 5 & 3 \\
\hline Eurema sp. & 7 & 3 & 2 & 0 & 2 & 1 \\
\hline Nepheronia thalassina & 8 & 11 & 3 & 4 & 15 & 2 \\
\hline Nepheronia argia & 7 & 4 & 3 & 5 & 6 & 2 \\
\hline Leptosia alcesta & 0 & 1 & 1 & 3 & 0 & 0 \\
\hline Leptosia medusa & 0 & 3 & 0 & 0 & 0 & 0 \\
\hline Leptosia sp. & 0 & 2 & 0 & 0 & 0 & 0 \\
\hline Appias phaola & 2 & 1 & 2 & 1 & 0 & 2 \\
\hline Dixeia astarte & 3 & 1 & 2 & 2 & 0 & 3 \\
\hline Colotis erone & 2 & 3 & 0 & 0 & 2 & 2 \\
\hline Colotis danae & 0 & 2 & 0 & 1 & 1 & 0 \\
\hline Colotis euippe & 5 & 3 & 4 & 3 & 2 & 3 \\
\hline
\end{tabular}




\section{Continued}

\begin{tabular}{|c|c|c|c|c|c|c|}
\hline Colotis antevippe & 7 & 8 & 8 & 5 & 3 & 5 \\
\hline Colotis eucharis & 1 & 3 & 2 & 0 & 1 & 0 \\
\hline Belenois calypso & 11 & 5 & 12 & 6 & 1 & 3 \\
\hline Belenois creona & 6 & 4 & 7 & 3 & 2 & 1 \\
\hline Belenois theora & 3 & 2 & 0 & 1 & 0 & 2 \\
\hline Belenois gidica & 0 & 1 & 1 & 1 & 3 & 1 \\
\hline Belenois aurota & 8 & 2 & 9 & 3 & 2 & 3 \\
\hline Belenois theuszi & 0 & 1 & 4 & 0 & 0 & 2 \\
\hline Catopsilia florella & 21 & 15 & 56 & 10 & 6 & 8 \\
\hline Mylothris chloris & 6 & 5 & 9 & 2 & 3 & 2 \\
\hline Mylothris poppea & 7 & 1 & 8 & 4 & 5 & 4 \\
\hline Mylothris sp. & 6 & 2 & 4 & 3 & 1 & 2 \\
\hline Mylothris rhodope & 5 & 1 & 9 & 2 & 0 & 1 \\
\hline Charaxes varanes & 1 & 3 & 2 & 0 & 1 & 1 \\
\hline Charaxes protoclea & 2 & 1 & 0 & 1 & 0 & 0 \\
\hline Charaxes etheocles & 0 & 1 & 1 & 0 & 0 & 0 \\
\hline Charaxes pelias & 1 & 2 & 0 & 0 & 1 & 0 \\
\hline Charaxes brutus & 0 & 1 & 4 & 0 & 0 & 0 \\
\hline Charaxes tiridates & 0 & 2 & 1 & 1 & 0 & 0 \\
\hline Charaxes cynthia & 2 & 4 & 2 & 2 & 1 & 0 \\
\hline Charaxes zelica & 0 & 3 & 1 & 0 & 1 & 0 \\
\hline Castalius carana & 6 & 22 & 5 & 2 & 4 & 0 \\
\hline Meza indusiata & 2 & 7 & 10 & 0 & 1 & 0 \\
\hline Euchrysops albistriata & 24 & 108 & 210 & 14 & 22 & 9 \\
\hline Euchrysops malathana & 8 & 9 & 16 & 7 & 5 & 2 \\
\hline Euchrysops barkeri & 9 & 27 & 31 & 6 & 4 & 0 \\
\hline Euchrysops osiris & 5 & 21 & 27 & 2 & 3 & 0 \\
\hline Anthene lunulata & 5 & 18 & 15 & 2 & 6 & 2 \\
\hline Anthene locuples & 3 & 12 & 8 & 2 & 1 & 0 \\
\hline Anthene staudingeri & 6 & 15 & 9 & 3 & 0 & 1 \\
\hline Lepidochrysops parsimon & 10 & 19 & 18 & 2 & 3 & 1 \\
\hline Lepidochrysops sp. & 14 & 23 & 25 & 1 & 4 & 2 \\
\hline Osmodes osmodes & 0 & 2 & 4 & 0 & 0 & 1 \\
\hline Spalgis lemolea & 1 & 3 & 0 & 2 & 0 & 0 \\
\hline Pyrrhiades lucagus & 0 & 6 & 3 & 3 & 2 & 2 \\
\hline Borbo perobscura & 1 & 7 & 0 & 0 & 0 & 1 \\
\hline
\end{tabular}


Table 2. Calculation of suite of diversity indices and richness estimators for butterfly species collected from the two sites at Kogyae Strict Nature Reserve, Dagomba (ED) and Oku (EO) for the three sampling seasons.

\begin{tabular}{cccccccccc}
\hline Sample & $S$ & $N$ & $d$ & $J^{\prime}$ & $H^{\prime}$ (loge) & ACE & Chao 1 & Jack knife 1 & Bootstrap \\
\hline ED1 & 63 & 312 & 10.8 & 0.9277 & 3.844 & 80.97 & 80.07 & 69.86 & 69.86 \\
ED2 & 106 & 739 & 15.9 & 0.842 & 3.927 & 97.59 & 98.73 & 108.12 & 99.12 \\
ED3 & 83 & 807 & 12.25 & 0.7524 & 3.325 & 99.84 & 100.32 & 109.99 & 103.4 \\
EO1 & 63 & 164 & 12.16 & 0.9377 & 3.885 & 106.07 & 106.02 & 117.05 & 110.61 \\
EO2 & 58 & 277 & 10.14 & 0.8289 & 3.366 & 106.84 & 106.41 & 115.99 & 111.24 \\
EO3 & 62 & 160 & 12.02 & 0.9242 & 3.814 & 107.89 & 107.6 & 116.17 & 112.08 \\
\hline
\end{tabular}

ED: Dagomba; EO: Oku; 1: September; 2: January; 3: June; S: species richness; $N$ : number of individuals; $d$ : Margalef's index; $J$ ': Pielou’s evenness index; $H^{\prime}$ : Shannon-Wiener index; ACE: Abundance coverage-based estimator.

orded for Dagomba in June was as a result of the dominance of species like Hammanumida daedalus and E. albistriata which made up about $32 \%$ of the total species abundance in June. The computed diversity indices are complemented by the species richness estimators within the study area. These estimators emphasize the representativeness of sampling effort in this study and hence indicated by the species accumulation curve (Figure 1). Abundance-based Coverage estimator (ACE) gives the estimated species richness expected using additional information based on species with 10 or fewer individuals in the sample based on the observed species abundance. To reduce bias, the first order Jack Knife and Bootstrap estimators of species diversity are also computed.

A dendogram of species population structure for the two sites at Kogyae for September (2011), January and June (2012) was computed (Figure 2) to compare the butterfly communities between the sites. Below a brayCurtis similarity of $85 \%$, the butterfly species population structure could be considered to be homogenous. Homogeneity begins to break down first around $88 \%$ with the formation of two clusters of species communities, ED2 and ED3 forming one cluster and ED1, EO2, EO1 and EO3 forming another. The clusters show that butterfly species composition was similar for Dagomba during the January and June sampling whiles species composition for Oku was somewhat similar for all three sampling seasons.

Figure 3 is a dendrogram showing the relationships between butterfly species occurring at Kogyae that contributed abundance of 20 or more using group-average linking on Bray-Curtis species similarity from fourth root transformed abundance data. Two main clusters are evident below a Bray-Curtis similarity of $90 \%$ with the other species somewhat loosely aggregated below a bray Curtis of $75 \%$.

\subsection{Odonata}

A total of 268 odonates belonging to three families and 20 species were recorded from KSNR, comprising of 16 species from the family Libellulidae, three species from the family Coenagrioniidae and one species from the family Calopterygidae. 198 individuals belonging to 17 species were recorded at Dagomba whilst 70 species belonging to 11 species were recorded at Oku. Dagomba (ED2) recorded the highest abundance $(N=82)$ as well as species numbers $(S=14)$ of dragonflies during the January sampling corroborated by the highest Shannon Weiner $H^{\prime}$ of 2.41, whiles the lowest species numbers $(S=6)$ and richness $(d=1.6)$ were recorded at Oku (EO3) during the June sampling. Dagomba (ED1) had a relatively low evenness during the September sampling (Table 3).

\subsection{Other Insects}

Apart from the butterflies and dragonflies, another 5688 insects belonging to 133 families (Table 4) from 21 insect orders were recorded from the traps. The Hymenopterans were the most abundant (3229) followed by the Dipterans (1543) which was also the most diverse with 39 families, and the Coleopterans with 21 families.

Diversity indices were calculated for the total of 133 insect families caught in the traps (Table 5). Dagomba (ED2) recorded the highest abundance of insects $(N=2622)$ with a relatively high family number $(S)$ of 84 in the dry season and the highest family diversity $(H=3.12)$ at the end of the rainy season in September. Oku 


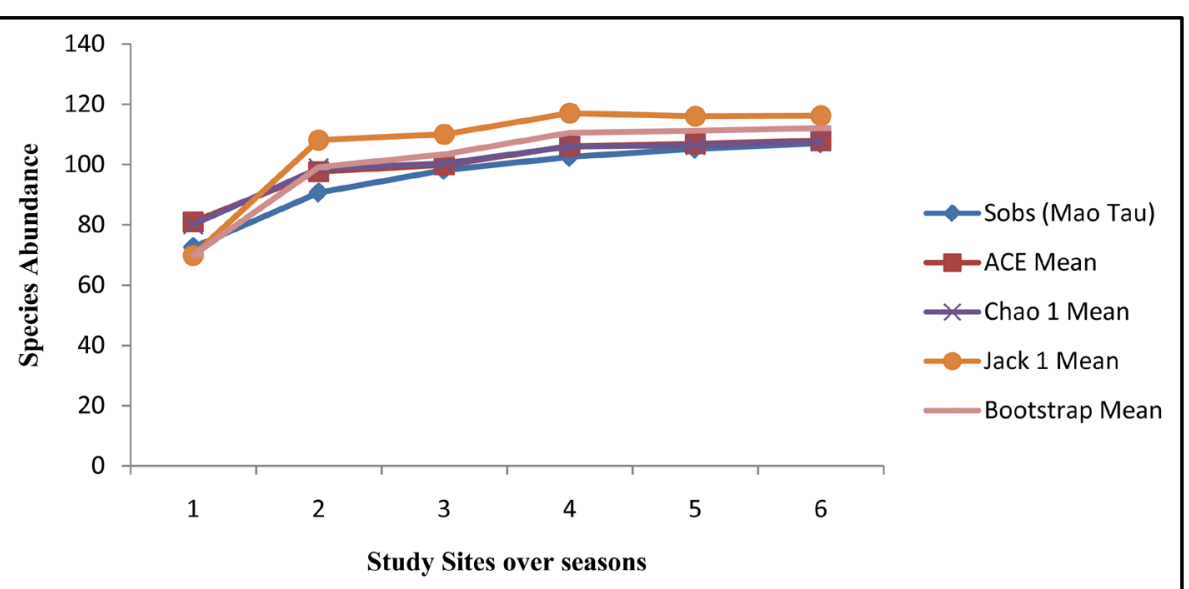

Figure 1. Species accumulation curve for butterfly assemblages within the Kogyae Strict Nature Reserve. Average species richness is based on 100 randomizations (1: ED1, 2: ED2, 3: ED3, 4: EO1, 5: EO2, 6: EO3).

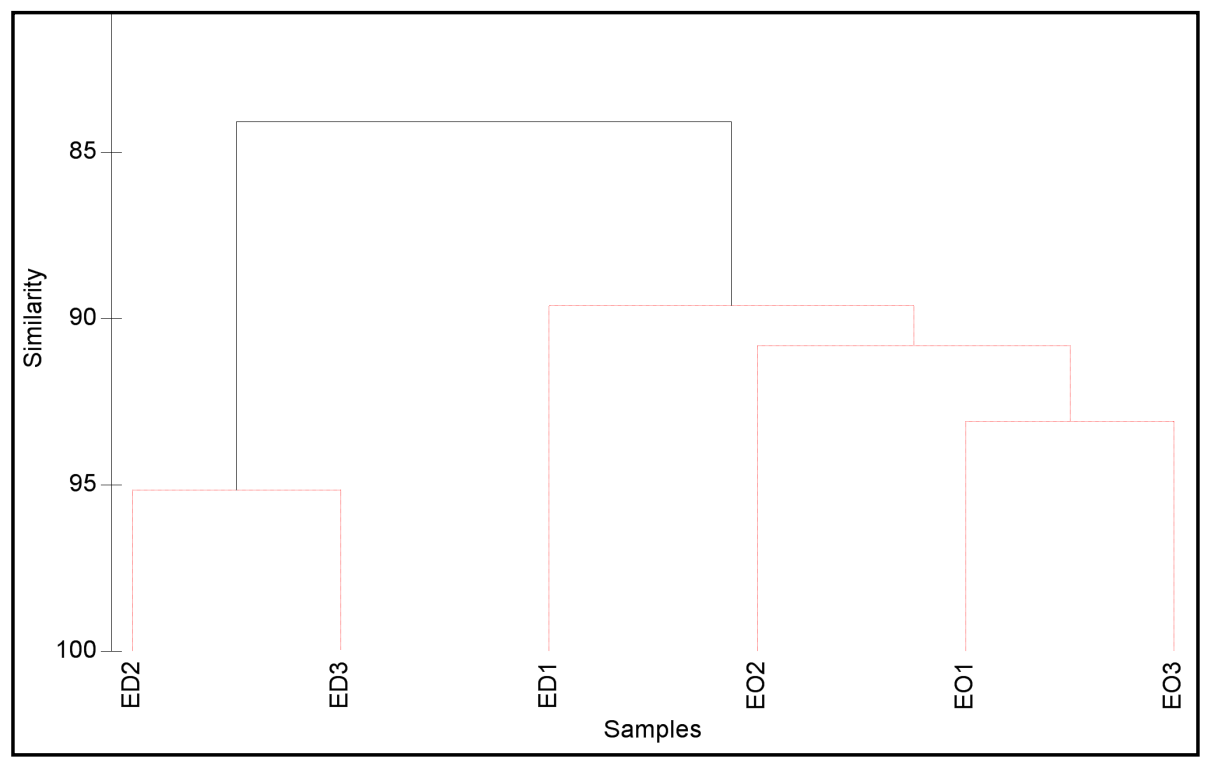

ED: Dagomba; EO: Oku; 1: July; 2: January; 3: June.

Figure 2. Dendrogram of butterfly species from the two sites at Kogyae Strict Nature Reserve in September (2011), January (2012) and June (2012). The cluster analysis was performed using group average linking on Bray Curtis species similarity from fourth root transformed abundance data.

(EO3) in June recorded the highest family richness $(d=13.45)$ during the wet season. The lowest abundance $(N$ $=567)$ and total family numbers $(S=66)$ corroborated by the lowest family richness $(d=10.25)$ were recorded for Oku (EO1) in September.

A cluster analysis using Bray Curtis similarity was performed for all the insects collected from the traps at both sites for the three sampling seasons (Figure 4). The cluster analysis revealed two distinct clusters/groupings around a Bray-Curtis similarity of 70\% (Figure 4) with homogeneity below a Bray-Curtis of around 65\%. Distribution of insects at both Dagomba and Oku were very similar for both the end of the wet and dry seasons since these two sampling seasons cluster together for both sites. Insect community distribution was also quite similar for both sites during the rainy season (Figure 4).

The insect order abundances were averaged and similarity analyses were performed on all 5688 insects from the 21 orders collected from the traps over the entire sampling period. The cluster analysis revealed two distinct 


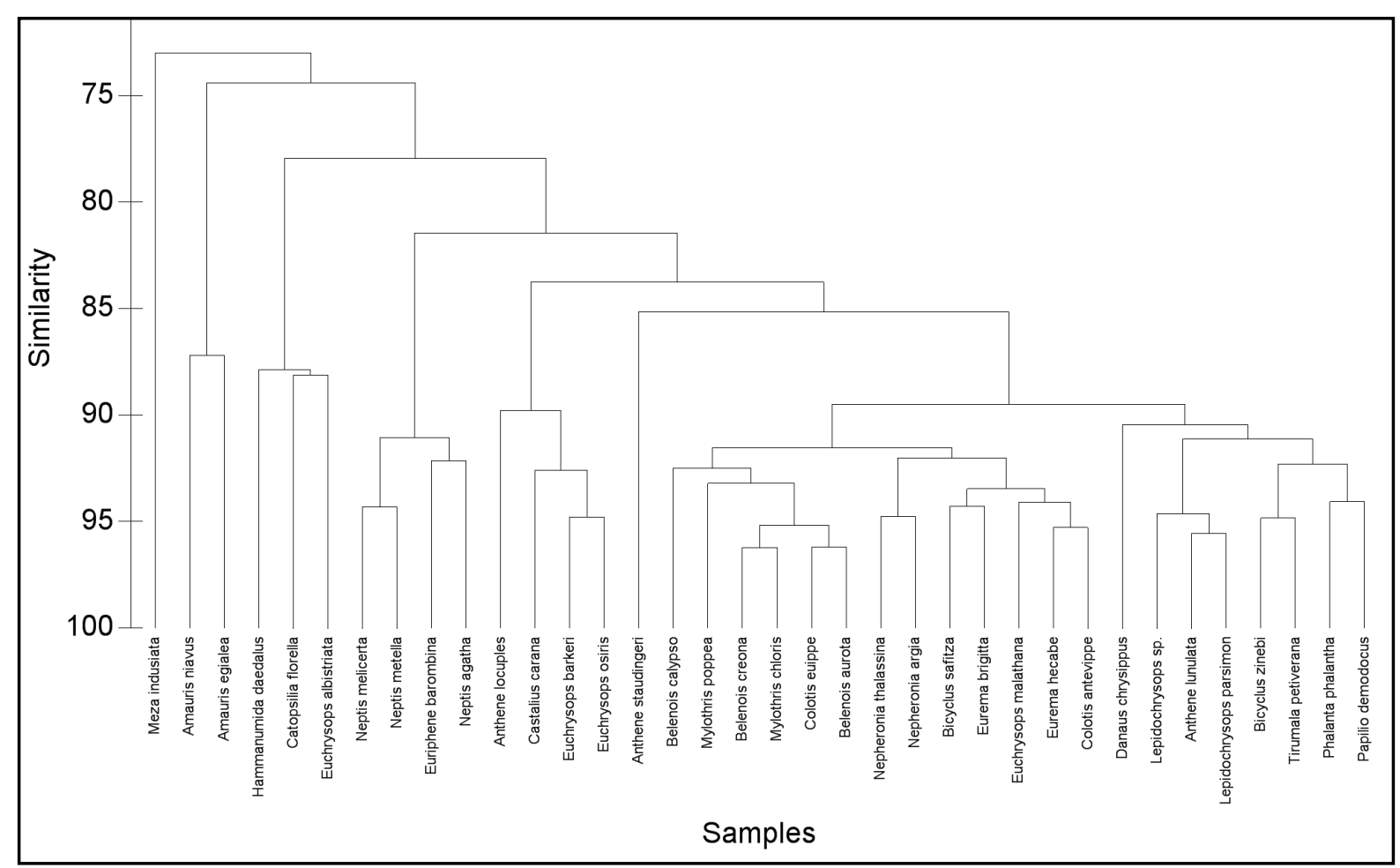

Figure 3. Dendrogram of butterfly species occurring at Kogyae Strict Nature Reserve using group average linking on Bray Curtis species similarity from fourth root transformed abundance data. The analysis was performed on species contributing abundance of 20 or more.

Table 3. Calculation of suites of diversity indices for Odonata collected from two sites Dagomba (ED) and Oku (EO) at Kogyae Strict Nature Reserve.

\begin{tabular}{cccccc}
\hline Sites & $S$ & $N$ & $d$ & $J^{\prime}$ & $H^{\prime}\left(\log _{e}\right)$ \\
\hline EO1 & 9 & 35 & 2.25 & 0.8974 & 1.972 \\
ED1 & 13 & 67 & 2.854 & 0.7376 & 1.892 \\
EO2 & 8 & 16 & 2.525 & 0.9685 & 2.014 \\
ED2 & 14 & 82 & 2.95 & 0.9155 & 2.416 \\
EO3 & 6 & 19 & 1.698 & 0.8281 & 1.484 \\
ED3 & 13 & 49 & 3.083 & 0.8466 & 2.172 \\
\hline
\end{tabular}

ED: Dagomba; EO: Oku; 1: September; 2: January; 3: June; S: species richness; N: number of individuals; $d$ : Margalef's index; $J^{\prime}$ : Pielou's evenness index; $H^{\prime}$ : Shannon-Wiener index.

clusters/groupings above a Bray-Curtis similarity of 30\% with homogeneity below a Bray-Curtis similarity of 30\% indicating a high level of dissimilarity in distribution (Figure 5). These main groupings further break down above a Bray-Curtis similarity of $70 \%$ into more or less four smaller groupings of more closely associated orders.

\section{Discussion}

\subsection{Butterflies}

Reference [7] estimated that 163 species of butterflies can be found at Kogyae but our study recorded 107 species from nine families of which 83 species were common to both Dagomba and Oku whiles 24 were unique to Dagomba. There were no species unique to Oku. Of the 24 species unique to Dagomba, more than half (17) 
Table 4. Insect families recorded within the Kogyae Strict Nature Reserve.

\begin{tabular}{|c|c|c|c|c|c|c|}
\hline \multirow{2}{*}{ FAMILY } & \multicolumn{3}{|c|}{ DAGOMBA } & \multicolumn{3}{|c|}{ OKU VILLAGE } \\
\hline & September & January & June & September & January & June \\
\hline Gryllidae & 2 & 8 & 20 & 10 & 11 & 16 \\
\hline Acrididae & 3 & 1 & 11 & 1 & 4 & 13 \\
\hline Tettigonidae & 1 & 3 & 3 & 2 & 1 & 6 \\
\hline Tridactylidae & 0 & 1 & 5 & 2 & 2 & 0 \\
\hline Tetrigidae & 2 & 8 & 3 & 1 & 12 & 1 \\
\hline Formicidae & 124 & 427 & 67 & 152 & 68 & 279 \\
\hline Apidae & 3 & 18 & 2 & 0 & 123 & 8 \\
\hline Evaniidae & 1 & 2 & 7 & 0 & 5 & 5 \\
\hline Sphecidae & 3 & 3 & 1 & 1 & 14 & 2 \\
\hline Vespidae & 2 & 9 & 4 & 1 & 27 & 6 \\
\hline Braconidae & 4 & 8 & 7 & 13 & 28 & 4 \\
\hline Ichneumonidae & 5 & 7 & 2 & 2 & 9 & 6 \\
\hline Scelionidae & 1 & 2 & 1 & 3 & 9 & 0 \\
\hline Halictidae & 18 & 986 & 25 & 15 & 643 & 7 \\
\hline Pompylidae & 0 & 2 & 3 & 0 & 4 & 4 \\
\hline Chalcididae & 1 & 7 & 0 & 0 & 8 & 1 \\
\hline Gasteruptidae & 2 & 0 & 0 & 1 & 2 & 0 \\
\hline Scoliidae & 1 & 0 & 0 & 0 & 3 & 0 \\
\hline Chrisididae & 1 & 2 & 1 & 0 & 3 & 0 \\
\hline Eulophidae & 0 & 2 & 0 & 1 & 5 & 0 \\
\hline Tiphiidae & 2 & 1 & 0 & 0 & 1 & 0 \\
\hline Platygastridae & 0 & 1 & 0 & 0 & 0 & 1 \\
\hline Blattidae & 17 & 23 & 2 & 2 & 9 & 5 \\
\hline Mantidae & 1 & 2 & 1 & 0 & 3 & 3 \\
\hline Noctuidae & 5 & 9 & 1 & 1 & 3 & 12 \\
\hline Arctidae & 2 & 8 & 3 & 0 & 9 & 4 \\
\hline Satyridae & 13 & 12 & 1 & 2 & 15 & 3 \\
\hline Pieridae & 0 & 1 & 1 & 0 & 0 & 1 \\
\hline Sphengidae & 1 & 0 & 0 & 0 & 1 & 0 \\
\hline Charaxidae & 0 & 0 & 2 & 0 & 3 & 1 \\
\hline Lycaenidae & 0 & 4 & 1 & 0 & 5 & 0 \\
\hline Acraeidae & 0 & 2 & 2 & 0 & 1 & 0 \\
\hline Nymphalidae & 0 & 1 & 0 & 1 & 2 & 0 \\
\hline Hesperidae & 0 & 3 & 1 & 0 & 0 & 1 \\
\hline
\end{tabular}




\section{Continued}

\begin{tabular}{|c|c|c|c|c|c|c|}
\hline Termitidae & 0 & 4 & 2 & 2 & 0 & 3 \\
\hline Cercopidae & 12 & 9 & 6 & 2 & 10 & 8 \\
\hline Aleyrodidae & 0 & 0 & 0 & 0 & 3 & 0 \\
\hline Aphididae & 1 & 0 & 0 & 0 & 0 & 1 \\
\hline Fulgoridae & 0 & 1 & 0 & 0 & 0 & 0 \\
\hline Cicadellidae & 15 & 12 & 11 & 3 & 10 & 9 \\
\hline Delphacidae & 0 & 2 & 0 & 0 & 1 & 3 \\
\hline Membracidae & 0 & 0 & 2 & 0 & 3 & 1 \\
\hline Acanalonidae & 0 & 0 & 0 & 0 & 0 & 1 \\
\hline Derbidae & 0 & 1 & 0 & 0 & 0 & 0 \\
\hline Psyllidae & 1 & 0 & 1 & 4 & 0 & 2 \\
\hline Isotomidae & 23 & 14 & 20 & 3 & 15 & 17 \\
\hline Calliphoridae & 2 & 3 & 2 & 13 & 8 & 1 \\
\hline Sepsidae & 3 & 0 & 2 & 0 & 0 & 1 \\
\hline Tachinidae & 3 & 12 & 5 & 12 & 11 & 8 \\
\hline Sarcophagidae & 1 & 2 & 5 & 2 & 3 & 5 \\
\hline Culicidae & 8 & 12 & 6 & 2 & 16 & 12 \\
\hline Muscidae & 4 & 6 & 4 & 8 & 9 & 5 \\
\hline Tephritidae & 1 & 0 & 3 & 3 & 4 & 0 \\
\hline Drossophilidae & 5 & 8 & 27 & 3 & 48 & 629 \\
\hline Phoridae & 41 & 15 & 33 & 10 & 12 & 11 \\
\hline Tipulidae & 2 & 0 & 0 & 2 & 1 & 4 \\
\hline Asilidae & 0 & 1 & 1 & 1 & 3 & 2 \\
\hline Diopsidae & 0 & 1 & 2 & 0 & 8 & 18 \\
\hline Dolichopodidae & 5 & 2 & 1 & 3 & 4 & 3 \\
\hline Syrrphidae & 3 & 8 & 2 & 2 & 10 & 9 \\
\hline Rhagionidae & 0 & 0 & 4 & 0 & 0 & 2 \\
\hline Stratiomyiidae & 0 & 2 & 1 & 0 & 0 & 1 \\
\hline Bombylidae & 0 & 0 & 0 & 0 & 5 & 1 \\
\hline Tabanidae & 1 & 0 & 2 & 0 & 2 & 5 \\
\hline Glossinidae & 0 & 5 & 8 & 6 & 24 & 14 \\
\hline Simulidae & 15 & 10 & 23 & 8 & 7 & 11 \\
\hline Mycetophilidae & 0 & 2 & 19 & 0 & 1 & 2 \\
\hline Sciaridae & 0 & 3 & 4 & 0 & 1 & 1 \\
\hline Chironomidae & 2 & 0 & 0 & 2 & 1 & 8 \\
\hline Sciomyzidae & 2 & 1 & 0 & 0 & 2 & 1 \\
\hline
\end{tabular}




\section{Continued}

\begin{tabular}{|c|c|c|c|c|c|c|}
\hline Chloropidae & 1 & 2 & 0 & 0 & 1 & 0 \\
\hline Pipunculidae & 1 & 0 & 0 & 0 & 2 & 0 \\
\hline Ceratopogonidae & 2 & 0 & 19 & 4 & 2 & 3 \\
\hline Conopidae & 0 & 1 & 6 & 3 & 0 & 0 \\
\hline Lauxanidae & 2 & 0 & 14 & 0 & 1 & 3 \\
\hline Cecidomyiidae & 0 & 0 & 7 & 0 & 2 & 1 \\
\hline Therevidae & 3 & 2 & 2 & 0 & 2 & 0 \\
\hline Platypezidae & 0 & 0 & 7 & 0 & 3 & 0 \\
\hline Trichoceridae & 0 & 1 & 6 & 0 & 0 & 3 \\
\hline Acroceridae & 0 & 2 & 4 & 0 & 1 & 0 \\
\hline Empididae & 2 & 2 & 19 & 3 & 5 & 2 \\
\hline Mycetophagidae & 0 & 0 & 0 & 0 & 0 & 4 \\
\hline Otitidae & 0 & 0 & 2 & 0 & 4 & 3 \\
\hline Anthomyiidae & 0 & 2 & 13 & 0 & 3 & 3 \\
\hline Bibionidae & 0 & 0 & 2 & 0 & 4 & 1 \\
\hline Chrysomelidae & 5 & 3 & 7 & 3 & 4 & 2 \\
\hline Carabidae & 0 & 0 & 1 & 6 & 0 & 2 \\
\hline Tenebrionidae & 2 & 0 & 0 & 1 & 0 & 0 \\
\hline Scarabaeidae & 1 & 0 & 1 & 7 & 2 & 1 \\
\hline Mordellidae & 5 & 1 & 1 & 1 & 0 & 0 \\
\hline Phalacridae & 0 & 0 & 1 & 2 & 0 & 2 \\
\hline Coccinellidae & 0 & 5 & 0 & 1 & 0 & 1 \\
\hline Curcujidae & 11 & 1 & 8 & 6 & 23 & 3 \\
\hline Lampyridae & 0 & 0 & 0 & 0 & 1 & 0 \\
\hline Staphylinidae & 12 & 4 & 2 & 6 & 24 & 4 \\
\hline Scolytidae & 1 & 0 & 0 & 0 & 0 & 0 \\
\hline Melandryidae & 1 & 0 & 0 & 0 & 1 & 0 \\
\hline Hydrophilidae & 2 & 0 & 0 & 0 & 0 & 0 \\
\hline Dyticidae & 1 & 0 & 0 & 0 & 0 & 0 \\
\hline Gyrinidae & 3 & 0 & 0 & 0 & 0 & 0 \\
\hline Meloidae & 2 & 0 & 0 & 0 & 0 & 0 \\
\hline Dermestidae & 1 & 0 & 0 & 0 & 1 & 0 \\
\hline Elmidae & 0 & 0 & 0 & 0 & 0 & 1 \\
\hline Scaphididae & 0 & 2 & 0 & 0 & 0 & 0 \\
\hline Curculionidae & 5 & 0 & 1 & 0 & 1 & 0 \\
\hline Cerambycidae & 0 & 1 & 0 & 0 & 0 & 1 \\
\hline
\end{tabular}




\section{Continued}

\begin{tabular}{|c|c|c|c|c|c|c|}
\hline Hydropsychidae & 3 & 4 & 1 & 2 & 2 & 3 \\
\hline Polycentropodidae & 0 & 1 & 0 & 1 & 2 & 1 \\
\hline Philopotamidae & 0 & 0 & 0 & 0 & 1 & 0 \\
\hline Thripidae & 6 & 2 & 6 & 1 & 9 & 2 \\
\hline Forfinculidae & 0 & 1 & 0 & 0 & 1 & 2 \\
\hline Labiidae & 0 & 2 & 0 & 0 & 1 & 0 \\
\hline Labiiduridae & 1 & 0 & 2 & 0 & 0 & 1 \\
\hline Carciniphoridae & 0 & 1 & 1 & 0 & 0 & 2 \\
\hline Libellulidae & 0 & 1 & 1 & 0 & 1 & 0 \\
\hline Coenagrionidae & 1 & 1 & 0 & 0 & 0 & 2 \\
\hline Pyrrhocoridae & 0 & 1 & 2 & 0 & 1 & 1 \\
\hline Coreidae & 0 & 0 & 0 & 0 & 1 & 0 \\
\hline Pentatomidae & 1 & 4 & 0 & 0 & 3 & 4 \\
\hline Reduvidae & 1 & 3 & 0 & 1 & 0 & 2 \\
\hline Largidae & 0 & 1 & 0 & 0 & 0 & 0 \\
\hline Cydnidae & 0 & 0 & 0 & 0 & 0 & 1 \\
\hline Thyreocoridae & 0 & 1 & 0 & 0 & 0 & 0 \\
\hline Ascalaphidae & 0 & 0 & 0 & 0 & 0 & 1 \\
\hline Myrmeleontidae & 0 & 0 & 1 & 0 & 1 & 0 \\
\hline Phasmatidae & 0 & 0 & 1 & 1 & 0 & 0 \\
\hline Perlidae & 0 & 0 & 0 & 0 & 0 & 5 \\
\hline Oligotomidae & 0 & 0 & 0 & 1 & 0 & 6 \\
\hline Ephemerellidae & 0 & 0 & 0 & 0 & 0 & 1 \\
\hline Ephemeridae & 0 & 0 & 0 & 0 & 0 & 2 \\
\hline Machilidae & 0 & 0 & 0 & 0 & 0 & 2 \\
\hline Lepismatidae & 0 & 0 & 0 & 0 & 0 & 1 \\
\hline Campodeidae & 0 & 0 & 0 & 0 & 0 & 1 \\
\hline
\end{tabular}

Table 5. Calculation of suites of diversity indices for insect families collected from two sites Dagomba (ED) and Oku (EO) at Kogyae Strict Nature Reserve.

\begin{tabular}{cccccc}
\hline Sites & $S$ & $N$ & $d$ & $J^{\prime}$ & $H^{\prime}\left(\log _{e}\right)$ \\
\hline ED1 & 80 & 848 & 11.72 & 0.7141 & 3.129 \\
ED2 & 84 & 2622 & 10.54 & 0.5409 & 2.396 \\
ED3 & 84 & 1430 & 11.42 & 0.6794 & 3.01 \\
EO1 & 66 & 567 & 10.25 & 0.7332 & 3.072 \\
EO2 & 95 & 1665 & 12.67 & 0.6411 & 2.92 \\
EO3 & 99 & 1457 & 13.45 & 0.5541 & 2.546 \\
\hline
\end{tabular}

ED: Dagomba; EO: Oku; 1: September; 2: January; 3: June; $S$ : species richness; $N$ : number of individuals; $d$ : Margalef's index; $J^{\prime}$ : Pielou's evenness index; $H^{\prime}$ : Shannon-Wiener index. 


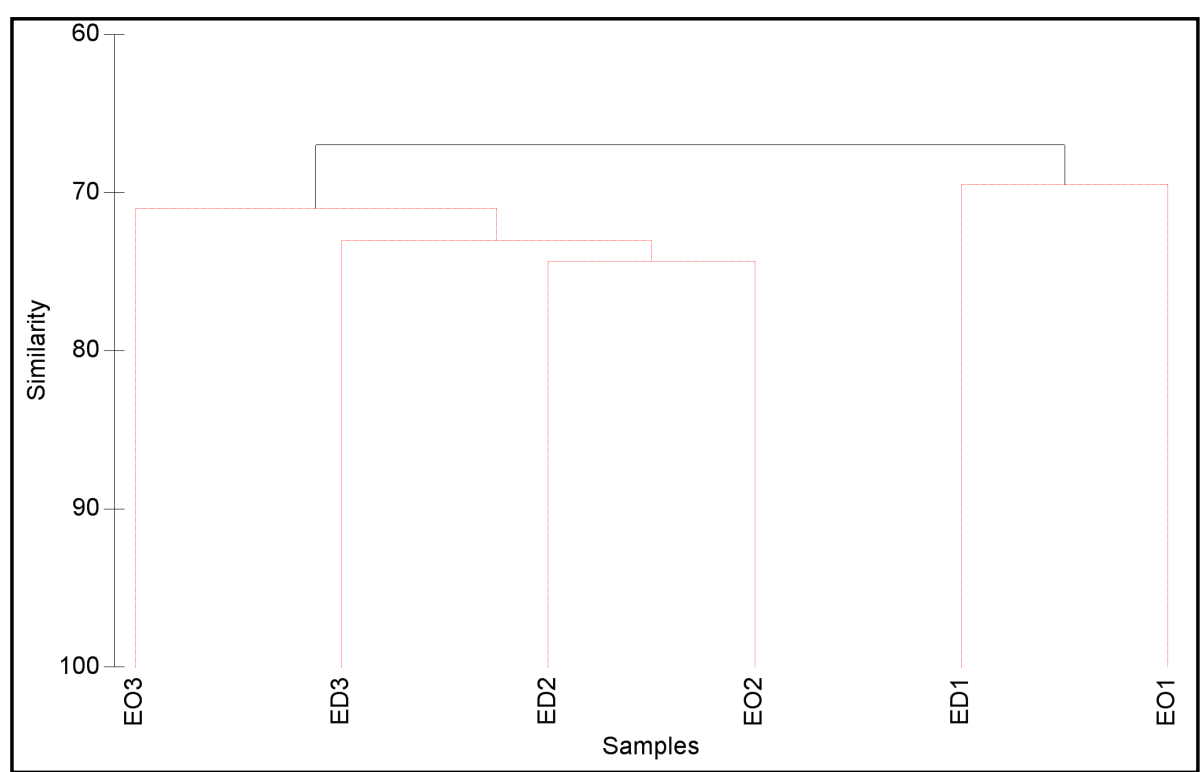

ED: Dagomba; EO: Oku; 1: July; 2: January; 3: June.

Figure 4. Dendrogram of all other insect species collected from two sites in Kogyae Strict Nature Reserve in September (2011), January (2012) and June (2012). The cluster analysis was performed using group average linking on Bray Curtis species similarity from fourth root transformed abundance data.

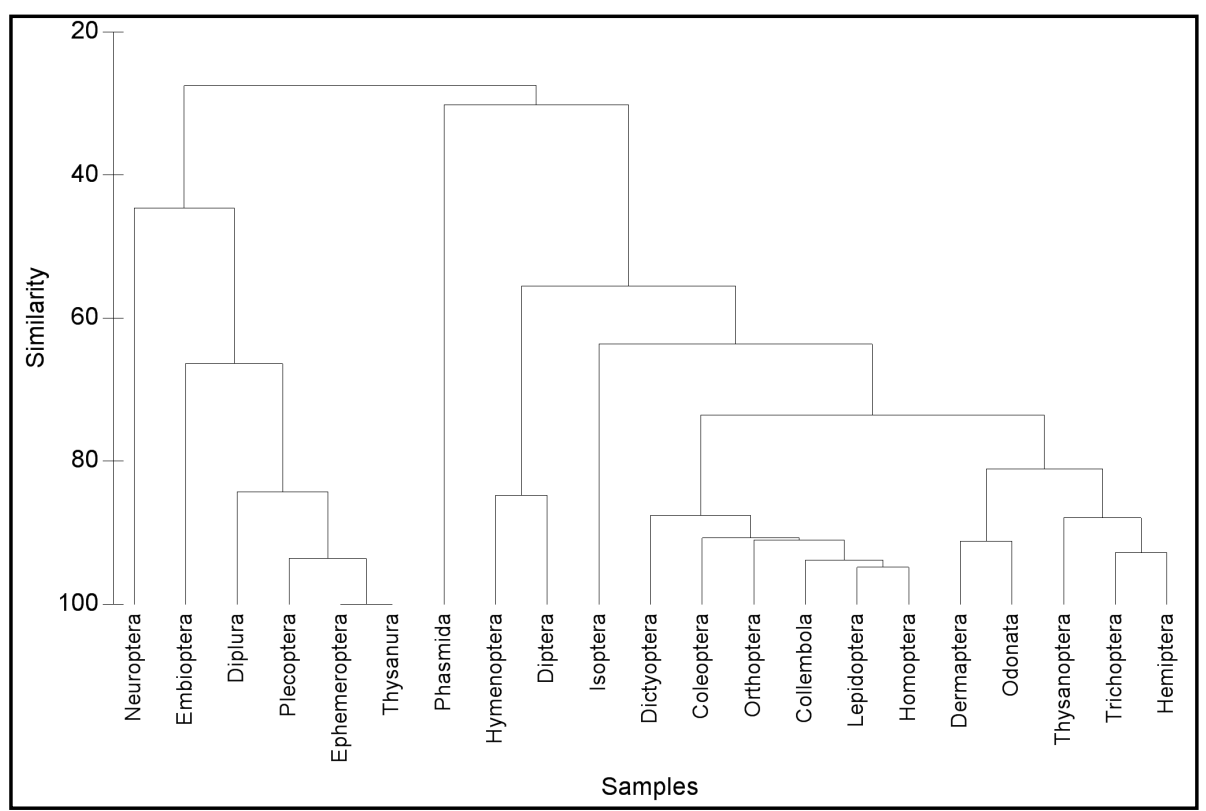

Figure 5. Dendrogram of all insect Orders collected from traps at the two sites at Kogyae Strict Nature Reserve (EO and ED) in September (2011), January (2012) and June (2012). The cluster analysis was performed using group average linking on Bray Curtis species similarity from fourth root transformed abundance data.

were species belonging to the family Nymphalidae such as Kalima rumia, Aterica galena and Gnophodes betsimena which are typical forest species. Most of these species were sampled along the riparian forest around the banks of the Afram River arm near the Asasebonso village. The most abundant species for the entire survey was Euchryssops albistriata which belong to the family Lycaenidae and are usually found aggregating around pools or puddles of water. Hamanumida daedalus, the second most abundant species, is typical of the Guinea Savan- 
nah open vegetation type found in the transitional zone of Ghana. They are usually seen almost hugging the floor along footpaths and roads in these areas. They were abundant at Dagomba which is more open. Typically grassland/open area species such as Papillio demodocus were observed in large numbers in the open areas of Dagomba in the dry season. Papillio nireus was relatively more abundant in the wet season around the riparian forest at Asasebonso. Danaus limniace and Belinois calypso can be found typically hovering around water bodies and so it is not surprising that they were more abundant around the river at Asasebonso. Species such as Nepheronia thalassina, Belinois calypso, Mylothris poppea, Catopsilla florella, Phalanta phalanta, though common to both sites, were more abundant at Dagomba a woodland savannah area with some rocky outcrops. These species are commonly found in African woodlands [32].

Species such as Bicyclus safitza and B. zinebi which are associated with woodland and forests throughout Africa [16] were common around the riparian forest at Dagomba. The increased presence of grass-feeding Satyrine species is an apparent sign of forest disturbance [33]. The dynamic between grasses and Satyrines signals the high potential of this group as indicators of forest condition, an attribute that could be beneficially exploited to help direct limited conservation resources in economically disadvantaged countries, e.g., to identify priority sites for formal protection or to steer restoration efforts [34].

The presence of deep forest species such as Charaxes brutus, Charaxes etheocles, Eurytela dryope, Ariadne enotera, and Euphedra eleus at Dagomba was not surprising giving the density of the riparian forest at Asasebonso. Even though there was riparian forest at Oku, it was not as dense and so fewer typical forest butterflies were recorded here.

Species such as Junonia oenone, J. terea, Papillio nireus, and P. demodocus are specialized in degraded habitats and open spaces and very few would ever be met within forest of good condition [32] [35]. Their presence around the riparian forests at both Oku and Dagomba is a clear indication of forest degradation (the result of the conversion of parts of the forest into farmlands). These species are known now to be much more common in West Africa than they ever were due to the widespread destruction and fragmentation of forest cover that has taken place in this part of Africa [19].

The high butterfly abundance ( $N=739$ ) as well as the high species numbers $(S=106)$ corroborated by the high Margalef index $(d)$ of 15.9 at Dagomba can be attributed to the mosaic kind of vegetation at this site which gives room for higher diversity of plant species. According to [36], the habitat heterogeneity hypothesis simply predicts that more arthropod species will occur where different forms and species of plants provide greater structural heterogeneity in the vegetation. Factors such as resource availability for adults and larval host-plants, behavioural traits and interaction with other species [37] may explain the increase in butterfly richness and diversity during the dry season at Dagomba. The high dominance of a few species such as Hammanumida daedalus, Euchrysops albistriata and Catopsilla florella accounted for the low evenness $\left(J^{\prime}=0.7524\right)$ recorded in June at Dagomba. During the dry season, most parts of the Reserve at Oku had been burnt down, so this would account for the low species numbers $(S=58)$. During the rainy season lower temperatures and less sunlight makes the butterflies less active hence the lowest abundance $(N=160)$ at this time. A study on butterflies of two sacred grooves in the Eastern Region of Ghana reported that butterflies were more speciose and diverse in the dry season most probably due to higher temperatures [35].

From the cluster analysis Colotis antevippe, Belinois calypso and Catopsilla florella for example cluster around a Bray Curtis similarity of 95\% (Figure 2). These species cluster together because they have similar behavioural traits and most feed on the same or related plant species. Papilio demodocus and Phalanta phalanta have similar distribution patterns and are closely associated and thus cluster around a Bray Curtis similarity of $93 \%$. Butterflies can serve-in a rough and ready manner-as proxies for terrestrial arthropod biodiversity as a whole.

\subsection{Odonata}

Odonata are relatively ever-present in freshwater wetlands, and they play an important role in the food chain of most ecosystems [38]. The adults are easily sampled and being susceptible to human disturbance, they constitute good candidates for wetlands assessment [39] [40]. Dragonflies can serve as biological indicators of environmental health [40]-[44]. Vegetation covering, can lead to a decline in population of adult dragonflies [45] or, in contrast, cause an increase in the species diversity [40] [42].

All the Odonata species recorded at KSNR are widespread species and therefore classified as of least concern 
[46]. The presence of species such as Pantala flavescens and Tramea basilaris indicate open areas or nearness to open areas whereas the presence of Phoan iridipennis (an African endemic) infer that the area it occurred in is adequately shaded, occurring especially in gallery forests [22]. Palpopleura lucia and Orthetrum julia are known to be widespread and very common species hence their high abundance in this study. The occurrence of a species like Bradynogpya strachani at Dagomba is a clear indication of the presence of human settlements within the KSNR as B. strachani typically settles on walls and boulders as mentioned by [22]; Oku harboured species like Chalcostephia flavifrons and Phaon iridipennis which, though open habitat species, prefer shaded forests.

\subsection{Other Insects}

Of the total of 5688 insects belonging 133 families and 21 orders recorded from the traps, the Hymenopterans were the most abundant (3229) whiles Diptera was the most diverse with 39 families followed by Coleoptera with 21 families. The Halictidae (sweat bees), Hymenoptera, were the most abundant family with over 1600 individuals recorded. Sweat bees are ground nesters forming their nests in clay soil or sandy banks of streams. A few species are attracted to sweat and may sometimes sting if disturbed [47]. They were recorded in large numbers along the banks of streams within the reserve. The Formicidae (ants), the second most abundant family thrive well in the warm moist weather conditions typically found in Kogyae. Most forage on the ground and low vegetation and nest in leaf litter, soil and rotten wood which were abundant in the sampling areas. Dipterans were mainly caught in the malaise traps set along the rivers and streams since many are aquatic and are trapped on emergence or as they come to oviposit close to or in the water.

The high insect diversity at both Dagomba $(H=3.12)$ and Oku $(H=3.07)$ in September at the end of the rainy season may be attributed to the fact that during this time of the year, there are ample food resources for the insects and the rainfall is not too heavy; heavy rainfall tends to sweep most insects away and destroy many of their food resources. The high dominance of Halictidae (sweat bees) may have accounted for the low evenness $(J=$ $0.54)$ recorded at Dagomba in the dry season. Family numbers $(S=99)$ as well richness $(d=13.45)$ were highest at Oku during the rainy season since most plants had recovered from the devastating effects of the bushfires that had occurred during the dry season and also due to habitat and food sources restoration.

A cluster analysis using Bray Curtis similarity was performed for all the insects collected from the traps at both sites for the three sampling seasons (Figure 4). Distribution of insects at both Dagomba and Oku were very similar for both the end of the wet and dry seasons. Interestingly, insect community distribution at Dagomba during the rainy season was closer to the distribution during the dry season for both sites than it was to the distribution at Oku during the wet season (Figure 4). What this means is that insect distribution for sites in the same general area during the same sampling season is not significantly different. There was a distinct cluster of species communities for both Oku and Dagomba in September and January indicating similar species composition and distribution.

The cluster analysis revealed two distinct clusters/groupings above a Bray-Curtis similarity of $30 \%$ with homogeneity below a Bray-Curtis similarity of $30 \%$ indicating a high level of dissimilarity in distribution for the insect orders (Figure 5). These main groupings further break down above a Bray-Curtis similarity of $70 \%$ into more or less four smaller groupings of more closely associated orders. Orders such as Thysanura, Diplura, Plecoptera and Ephemeroptera clustered together above a Bray Curtis similarity of 90\% indicating close association considering their similar distributions and lifestyles. They can be found in moist decaying vegetation and habitats, especially around banks of rivers and streams. Diptera and Hymenoptera cluster together above a Bray Curtis similarity of $80 \%$ also indicating a high level of similarity in distribution. These two orders contributed to $84 \%$ of the entire insect collection from the traps at both sites.

\section{Conclusions}

This study has provided the first comprehensive inventory of insects of the Kogyae Strict Nature Reserve. Insect biodiversity at the Reserve was very high with 135 families of insects from 21 orders. A total of 107 species of butterflies from nine families were recorded. Both sites sampled were very rich in species with a somewhat even distribution though the numbers varied greatly depending on the type of vegetation and the amount of forest cover. Of the two sites sampled, the area around Dagomba village especially the riparian forest at Asasebonso recorded the highest diversity of insects at the end of the rainy season. Insect species composition and distribu- 
tion were similar for the two sampling sites at the end of the rainy season and during the dry season. The most abundant butterfly species for the entire survey was the Lycinid Euchryssops albistriata usually found aggregating around pools or puddles of water. Butterfly species composition was similar at Dagomba for the dry and wet seasons, whereas at Oku, species composition was similar at the peak and end of the wet season.

Though most Odonata recorded from this survey was widespread and open habitat species, it can be said that the KSNR is likely to hold much more species than those recorded in this study due to its many inaccessible water bodies. Also, the sighting of Chalcostephia flavifrons and Phaon iridipennis infers the existence of forested areas of appreciable quality.

Insects play a major role in maintaining the health of the ecosystems. They are mainly responsible for pollination of plants, serve as scavengers to clean up the systems of dead materials as well as contribute immensely to decomposition. Apart from the plants, they constitute a major source of food for birds, mammals, reptiles and other taxonomic groups of animals. Their demise will therefore result in the disruption of critical ecosystem services such as pollination and source of food.

\section{Acknowledgements}

This study was undertaken under the building capacity to meet the climate change challenge (B4C)-Ghana project, which is implemented by a consortium of three institutions, the University of Ghana (lead), the Centre for African Wetlands, and the Ghana Wildlife Society, with funding from the Open Society Foundations. The authors are grateful to the Open Society Foundations for the funds that made the study possible.

\section{References}

[1] Hagan, J.E. (1998) The Kogyae Strict Nature Reserve. The World Bank/WBI's CBNRM Initiative.

[2] Margules, C. and Pressey, R. (2000) Systematic Conservation Planning. Nature, 405, 243-253. http://dx.doi.org/10.1038/35012251

[3] Raina, S.K., Chauhan, T.P.S., Tayal, M.K., Pandey, R.K. and Mohan, R. (2011) Multiple Silkworm Cocoon Cropping in Jammu Division. Proceedings of the Workshop on Recent Trends in Development of Sericulture in Jammu and Kashmir, Jammu and Kashmir, 63-67

[4] Buchs, W. (2003) Biotic Indicators for Biodiversity and Sustainable Agriculture. Elsevier Science Ltd., Amsterdam, $560 \mathrm{p}$.

[5] Scholtz, C.H. and Mansell, M.W. (2009) Insect Biodiversity in the Afrotropical Region. In: Foottit, R. and Adler, P., Eds., Insect Biodiversity: Science and Society, Blackwell, 69-82.

[6] European Union (2010) Overview of Kogyae Strict Nat. http://bioval.jrc.ec.europa.eu/APAAT/pa_report.php/pa/1543

[7] Larsen, T.B. (2006) The Ghana Butterfly Fauna and Its Contribution to the Objectives of the Protected Areas System. WDSP Report No. 63, in Collaboration with IUCN.

[8] CI (2007) Conservation International (CI) 2007 Annual Report.

[9] UNEP-WCMC (2006) WDPA Consortium 2006 World Database on Protected Areas.

[10] Carter, D. (1998) Butterflies and Moths. Stoddart Publishing.

[11] Gullan, P.J. and Cranston, P.S. (2010) Insects: An Outline of Entomology. Chapman and Hall, London.

[12] Gullan, P.J. and Cranston, P.S. (2005) Insects: An Outline of Entomology. 3rd Edition, Blackwell Publishing Ltd., Hoboken, 505 p.

[13] Scholtz, C.H. and Holm, E. (1989) Insects of Southern Africa. Butterworths Professional Publishers Ltd.

[14] Chinery, M. (1993) Insects of Britain and Northern Europe. HarperCollins Publishers, New York.

[15] Chinery, M. (1995) Butterflies and Moths; Photoguide. HarperCollins, New York.

[16] Carcasson, R.H. (1981) Collins Handguide to the Butterflies of Africa. William Collins Sons \& Co. Ltd., Glasgow.

[17] Crowson, R.A. (1956) Coleoptera: Introduction and Key to Families. Handbooks for the Identification of British Insects, Vol. 4, Part 1.

[18] Belcastro, C. and Larsen, T.B. (2006) Butterflies as an Indicator Group for the Conservation Value of the Gola Forests in Sierra Leone. Report to the Gola Forest Conservation Concession Project.

[19] Larsen, T.B. (2005) Butterflies of West Africa. Apollo Books, Vol. 2, 596. 
[20] Miller, P.L. (1987) Dragonflies. Richmond Publishers.

[21] McGavin, G.C. (2002) Insects, Spiders and Other Terrestrial Arthropods. Dorling Kindersley Inc., London.

[22] Dijkstra, K.D.B. and Clausnitzer, V. (2013) The Dragonflies and Damselflies of Eastern Africa: Handbook for All Odonata from Sudan to Zimbabwe. Studies in Afrotropical Zoology, Belgian Royal Museum for Central Africa, Tervuren. (In Press)

[23] Oldroyd, H. (1970) Diptera: Introduction and Key to Families. Handbooks for the Identification of British Insects, Vol. 4, Part 1.

[24] Clarke, K.R. and Warwick, R.M. (2001) Change in Marine Communities: An Approach to Statistical Analysis and Interpretation. 2nd Edition, PRIMER-E, Plymouth, $172 \mathrm{p}$

[25] Clarke, K.R. and Gorley, R.N. (2006) PRIMER V6: User Manual/Tutorial. PRIMER-E, Plymouth, 192 p.

[26] Colwell, R.K. and Coddington, J.A. (1994) Estimating Terrestrial Biodiversity through Extrapolation. Philosophical Transactions of the Royal Society B, 345, 101-118. http://dx.doi.org/10.1098/rstb.1994.0091

[27] Colwell, R.K. (2000) Estimate S-Statistical Estimation of Species Richness and Shared Species from Samples. Version 6.

[28] Colwell, R.K., Mao, C.X. and Chang, J. (2004) Interpolation, Extrapolating and Comparing Incidence-Based Species Accumulation Curves. Ecology, 85, 2717-2727. http://dx.doi.org/10.1890/03-0557

[29] Magurran, A.E. (2010) Measuring Biological Diversity. Blackwell Publishing, Malden, 256 p.

[30] Marshall, J.C., Steward, A.L. and Harch, B.D. (2006) Taxonomic Resolution and Quantification of Freshwater Macroinvertebrate Samples from an Australian Dryland River: The Benefits and Costs of Using Species Abundance Data. Hydrobiologia, 572, 171-194. http://dx.doi.org/10.1007/s10750-005-9007-0

[31] Magurran, A.E. (2004) Measuring Biological Diversity. Blackwell Publishing, Oxford, 256 p.

[32] Kyerematen, R., Acquah-Lamptey, D., Owusu, E.H., Anderson, R.S. and Ntiamoa-Baidu Y. (2014) Insect Diversity of the Muni-Pomadze Ramsar Site: An Important Site for Biodiversity Conservation in Ghana. Journal of Insects, 2014 Article ID: 985684, 11 p. http://dx.doi.org/10.1155/2014/985684

[33] Larsen, T.B. (1994) The Butterflies of Ghana: Their Implications for Conservation and Sustainable Use. Report to IUCN and Department of Game and Wildlife.

[34] Bossart, J.L., Opuni-Frimpong, E., Kuudaar, S. and Nkrumah, E. (2006) Richness, Abundance, and Complementarity of Fruit-Feeding Butterfly Species in Relict Sacred Forests and Forest Reserves of Ghana. Biodiversity \& Conservation, 15, 333-359. http://dx.doi.org/10.1007/s10531-005-2574-6

[35] Nganso, B.T., Kyerematen, R. and Obeng-Ofori, D. (2012) Diversity and Abundance of Butterfly Species in the Abiriw and Odumante Sacred Groves in the Eastern Region of Ghana. Research in Zoology, 2, 38-46. http://dx.doi.org/10.5923/j.zoology.20120205.01

[36] Hart, D.D. and Horwitz, R.J. (1991) Habitat Diversity and the Species Area Relationship: Alternative Models and Tests. In: Bell, S.S., McCoy, E.D. and Mushinsky, H.R., Eds., Habitat Structure: The Physical Arrangement of Objects in Space, Chapman and Hall, London, 47-68.

[37] Pinheiro, C.E.G. and Ortiz, J.V.C. (1992) Communities of Fruit Feeding Butterflies along a Vegetation Gradient in Central Brazil. Journal of Biogeography, 19, 505-511. http://dx.doi.org/10.2307/2845769

[38] Vashishth, N., Joshi, P.C. and Singh, A. (2000) Odonata Community Dynamics in Rajaji National Park, India. Fraseria, 7, 21-25.

[39] Bried, J.T. (2005) Community and Conservation Ecology of Dragonfly and Damselfly Adults in Mississippi Wetlands. Thesis, Mississippi State University, Mississippi.

[40] Watson, J.A.L., Arthington, A.H. and Conrick, D.L. (1982) Effect of Sewage Effluent on Dragonflies (Odonata) of Bulimba Creek, Brisbane. Australian Journal of Marine and Freshwater Resources, 33, 517-528. http://dx.doi.org/10.1071/MF9820517

[41] Clark, T.E. and Samways, M.J. (1996) Dragonflies (Odonata) as Indicators of Biotype Quality in the Kruger National Park, South Africa. Journal of Applied Ecology, 33, 1001-1012. http://dx.doi.org/10.2307/2404681

[42] Stewart, D.A.B. and Samways, M.J. (1998) Conserving Dragonfly (Odoanta) Assemblages Relative to River Dynamics in an African Savanna Game Reserve. Conservation Biology, 12, 683-692. http://dx.doi.org/10.1046/j.1523-1739.1998.96465.x

[43] Clausnitzer, V. and Jödicke, R. (2004) Guardians of the Watershed. International Journal of Odonatology, 7, 111. http://dx.doi.org/10.1080/13887890.2004.9748202

[44] Acquah-Lamptey, D., Kyerematen, R. and Owusu, E.O. (2013) Using Odonates as Markers of the Environmental Health of Water and Its Land Related Ecotone. International Journal of Biodiversity and Conservation, 5, 761-769. 
[45] Moore, N.W. (1997) Dragonflies: Status Survey and Conservation Action Plan. IUCN/SSC Odonata Specialist Group, $27 \mathrm{p}$.

[46] IUCN (2013) The IUCN Red List of Threatened Species. Version 2013.2. http://www.iucnredlist.org

[47] Michener, C.D. (2007) Bees of the World. 2nd Edition, The Johns Hopkins University Press, Baltimore. 
Scientific Research Publishing (SCIRP) is one of the largest Open Access journal publishers. It is currently publishing more than 200 open access, online, peer-reviewed journals covering a wide range of academic disciplines. SCIRP serves the worldwide academic communities and contributes to the progress and application of science with its publication.

Other selected journals from SCIRP are listed as below. Submit your manuscript to us via either submit@scirp.org or Online Submission Portal.
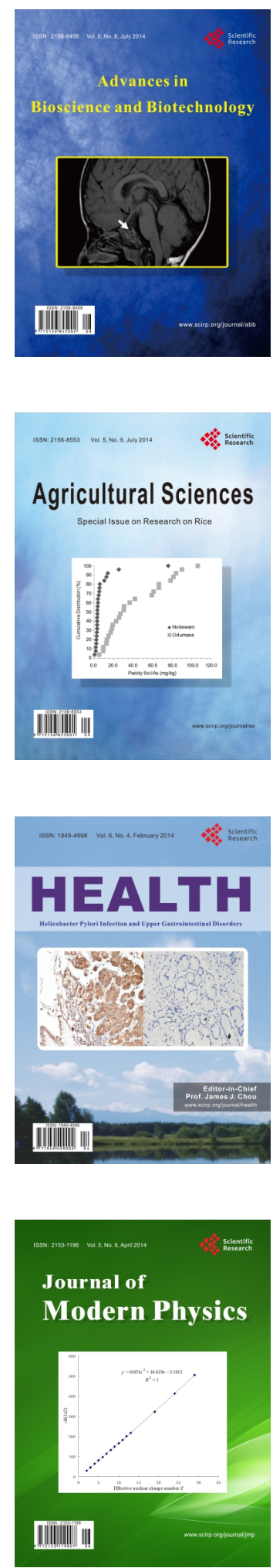
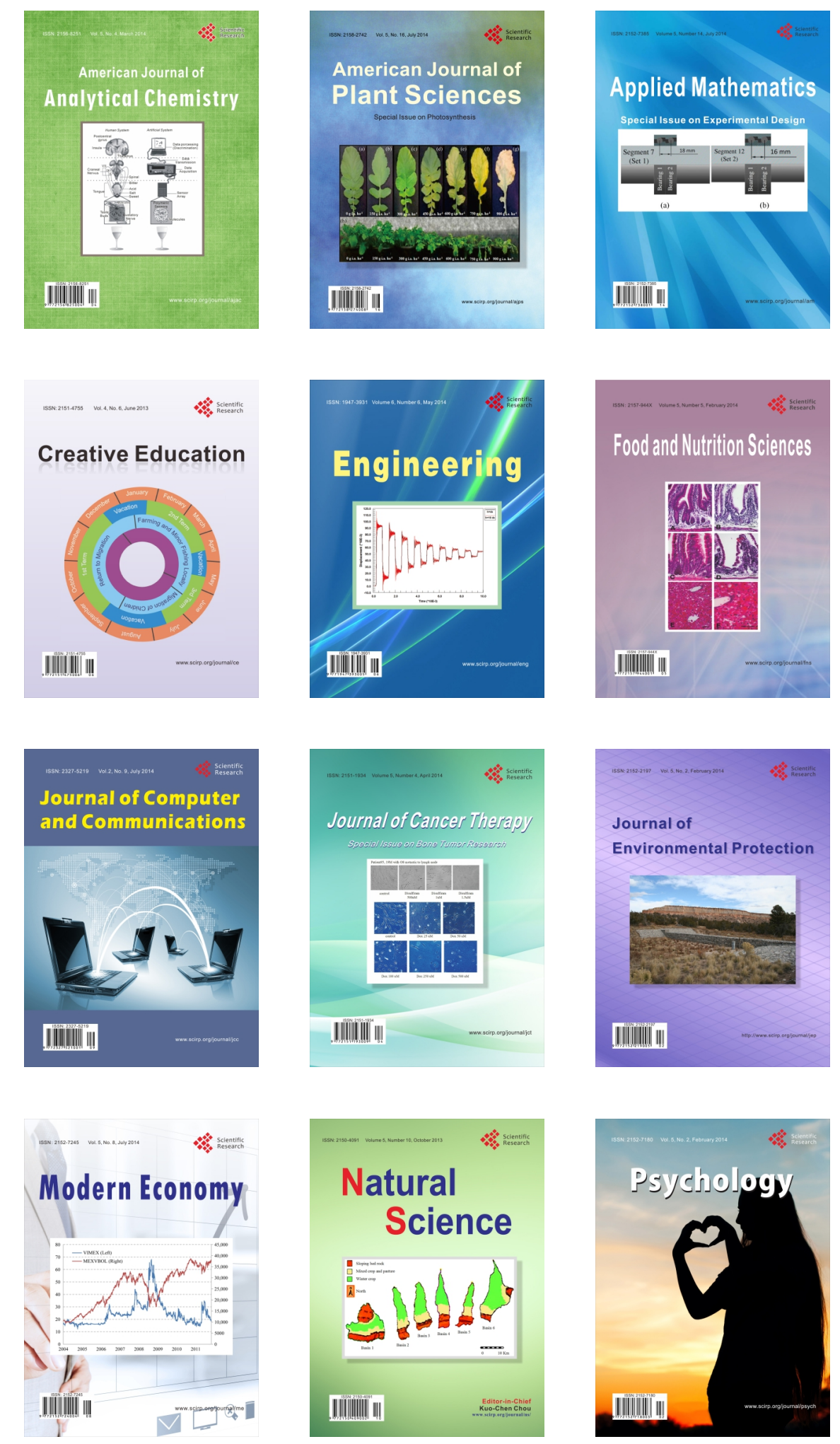